\title{
The Role of Cardio-Ankle Vascular Index as a Predictor of Mortality in Patients on Maintenance Hemodialysis
}

\author{
Kouichi Murakami ${ }^{1}$ \\ Emi Inayama' \\ Yukiko Itoh' \\ Shoji Tuchiya' \\ Masashi Iwasaki ${ }^{2}$ \\ Nobuko Tamura' \\ Toshihiko Suzuki ${ }^{3}$ \\ Noriko Iwai' \\ Junji Utino ${ }^{4}$ \\ Motoyuki Masai $\mathbb{1}^{4}$ \\ Daiji Nagayama $\mathbb{( I D}^{5,6}$ \\ Kohji Shirai ${ }^{4,6}$
}

'Seijinaki Mihama Narita Clinic, Narita City, Chiba, 286-004I, Japan; ${ }^{2}$ Seijinkai Mihama Katori Clinic, Katori City, Chiba, 287-004I, Japan; ${ }^{3}$ Seijinkai Mihama Sakura Clinic, Sakura City, Chiba, 285-084I, Japan; ${ }^{4}$ Seijinkai Mihama Hospital, Chiba City, Chiba, 26I-0013, Japan; ${ }^{5}$ Nagayama Clinic, Oyama City, Tochigi, 323-0032, Japan; ${ }^{6}$ Sakura Hospital, School of Medicine Toho University, Sakura City, Chiba, 285-874I, Japan
Correspondence: Daiji Nagayama Nagayama Clinic, 2-12-22, Tenjin-Cho, Oyama City, Tochigi, 323-0032, Japan $\mathrm{Tel} / \mathrm{Fax}+8 \mathrm{I}-285-22-0219$

Email deverlast9607I@gmail.com

\begin{abstract}
Aim: Mortality rate of maintenance hemodialysis patients is known to be high. Cardio-ankle vascular index (CAVI) is an index reflecting the proper stiffness of the arterial tree from the origin of the aorta to the ankle. We aimed to clarify the utility of CAVI as a predictor of mortality in hemodialysis patients. The roles of age and nutritional conditions on survival were also examined.
\end{abstract}

Methods: We followed 242 patients undergoing hemodialysis for 6 consecutive years. Data from 209 patients (mean age was $60 \pm 11$ years) excluding those with ankle-brachial index $<0.90$ were then analyzed. CAVI and heart to ankle pulse wave velocity (haPWV) were measured using Vasera 1500.

Results: Thirty-eight hemodialysis patients who died during the 6-year period had higher age, cardiothoracic ratio (CTR), CAVI, and haPWV, and lower diastolic blood pressure, albumin, phosphate, and calcium phosphate product. The Kaplan-Meier curves for cumulative survival among the tertile groups showed that the mortality rate was higher in the highest tertile (T3) compared to T1/T2 for both CAVI and haPWV. Receiver operating characteristic (ROC) analysis revealed that CAVI had better discriminatory power for all-cause mortality compared to haPWV. In the Cox-proportional hazards analyses, $1 \mathrm{SD}$ increase in both parameters contributed independently to all-cause mortality [CAVI: HR 1.595 (95\% CI 1.108-2.297), haPWV: HR 1.695 (95\% CI 1.185-2.425)], as well as age and CTR. Both parameters above the cut-offs estimated in the ROC analysis (CAVI $\geq 9.2$, haPWV $\geq 8.9$ ) also had independent contributions to mortality.

Conclusion: Through the 6 consecutive years of follow-up in 209 HD patients, increased CAVI might represent a major modifiable risk factor for all-cause mortality. Further research is needed to examine whether CAVI-lowering interventions contribute to improved prognosis.

Keywords: hemodialysis patient, mortality, cardio-ankle vascular index, phosphorus

\section{Introduction}

The mortality rate of maintenance hemodialysis (HD) patients is high, ${ }^{1}$ and numerous causes of death in HD patients have been suggested. ${ }^{2}$ Among them, the main cause of death has been reported to be associated with atherosclerotic diseases. ${ }^{3}$ To evaluate the degree of atherosclerosis noninvasively is not easy. Nevertheless, arterial stiffness is one of the candidate indexes reflecting the degree of arteriosclerosis, and pulse wave velocity (PWV) was used to evaluate the stiffness. ${ }^{4} \mathrm{El}$ Ghoul et $\mathrm{al}^{5}$ reported that carotid-femoral PWV (cfPWV) is a predictor of the cardiovascular diseases (CVD) events among HD patients. However, PWV is 
essentially dependent on blood pressure (BP) at the time of measurement, which has led to an overestimation of the role of hypertension in epidemiological studies. To overcome this problem, the cardio-ankle vascular index (CAVI) has been developed. ${ }^{6}$ CAVI reflects the stiffness of the whole arterial tree composing the aorta, femoral artery and tibial artery. This index was originally derived from the stiffness parameter $\beta$ proposed by Hayashi et $\mathrm{al}^{7}$ and Kawasaki et al. ${ }^{8}$ The theory of stiffness parameter $\beta$ was applied to a length of artery with the application of Bramwell-Hill's equation. ${ }^{9}$ Independency of CAVI from BP at measuring time was established both theoretically and experimentally. ${ }^{10,11}$

As for chronic kidney disease (CKD), several reports state that CAVI is negatively correlated with the estimated glomerular filtration rate. $^{12,13}$ Besides, CAVI showed a high value in HD patients. ${ }^{14,15}$ Furthermore, Sato et al reported that CAVI is a predictor of future CVD events based on the 6-year follow-up of 1003 patients with metabolic disorders. ${ }^{16}$ Kirigaya et al reported that CAVI was an independent long-term predictor of CVD death in patients with acute coronary syndrome. ${ }^{17}$ Recently, Itano et al reported that increased CAVI in adults without CKD may be a risk for future renal dysfunction. ${ }^{18}$ On the other hand, as for HD patients, Kato et al ${ }^{19}$ reported that brachialankle PWV (baPWV) is superior to CAVI as a predictor of CVD outcomes in 135 patients on regular HD in an observation period of 110 months. Against this background, the significance of CAVI as a predictor of mortality in HD patients has not been well established yet.

In this report, the relationship of CAVI with mortality rate was investigated by following 209 HD patients for 6 consecutive years of follow-up. Furthermore, several factors such as cardio-thoracic ratio (CTR), BP, glycated hemoglobin (HbAlc), serum phosphorus and albumin, which are known to be a risk for mortality, ${ }^{1,20}$ were also analyzed.

\section{Aim}

This study aimed to clarify the validity of CAVI as a predictor of all-cause mortality in HD patients. The other confounders were also examined.

\section{Methods}

\section{Subjects}

The initial subjects were 242 patients who received maintenance HD therapy at Seijinkai Mihama Narita Clinic and
Seijinkai Mihama Katori Clinic in 2009. Patients with ankle brachial index less than 0.90 were excluded, because a reliable CAVI value was not obtained. Finally, we analyzed archival data retrospectively on 209 HD patients for 6 consecutive years, collected from existing records. The mean age was $60 \pm 11$ (range 38-89) years old. The mean duration of HD therapy was $110 \pm 93$ months. The patients were treated with conventional in-center HD three times a week.

\section{Ethics Approval and Consent to Participate}

The protocol of the study was prepared in accordance with the Declaration of Helsinki, and this study was approved by the Ethics Committee in Seijinkai Mihama Hospital (No 21-001). Written informed consent was obtained in the form of opt-out.

\section{Factors Related to the Mortality}

As for the possible contributing factors for the mortality rates, sex, age, HD vintage, history of diabetes mellitus, hypertension, BP, CTR and heart to ankle PWV (haPWV) were analyzed. Height and body weight (dry weight) were measured, and body mass index (BMI) was calculated as follows: body weight ( $\mathrm{kg}$ ) divided by square of height $(\mathrm{m})$. Furthermore, biochemical laboratory levels, such as serum albumin, HbAlc, triglyceride (TG), high-density lipoprotein (HDL)-cholesterol, low-density lipoprotein (LDL)cholesterol, calcium, inorganic phosphorus and uric acid levels were included in the analysis. Calcium phosphate product (CPP) was calculated as an index of disturbed mineral metabolism. All these items were measured using the venous blood sample obtained at the beginning of HD therapy over a 2-day interval. The values used for analysis were the means of 6 months from the beginning.

\section{Measurement of CAVI}

CAVI was measured using VaSera1500 (Fukuda Denshi, Co., Ltd., Tokyo). ${ }^{6}$ The timing of measuring CAVI was from the first $30 \mathrm{~min}$ to $60 \mathrm{~min}$ during HD therapy, because the CAVI value of most patients is rather stable during this time period. Also, haPWV was obtained simultaneously by VaSera 1500 .

\section{Statistical Analysis}

The results are expressed as mean \pm standard deviation (SD). For comparison of two independent groups, all data 
were analyzed using Mann-Whitney $U$-test or Fisher's exact test. Pearson's product-moment correlation coefficient (r) was used to examine the relationship between clinical variables. Kaplan-Meier survival analysis was employed to estimate the differences of the time to allcause mortality between the groups. Additionally, covariate-adjusted survival curves were estimated from the Coxproportional hazards model. Sensitivity and specificity in arterial stiffness parameters with respect to all-cause mortality were analyzed using conventional receiver-operating -characteristic (ROC) curves. The ROC curves and Youden's J Index [ $\mathrm{J}$ is defined as the maximum of (sensitivity + specificity -1 ) to provide cut-off points in the ROC curve], ${ }^{21}$ were generated to evaluate the discriminatory powers and cut-off values of both arterial stiffness parameters for all-cause mortality. An area-under curve (AUC) reflecting the discriminatory power of two arterial stiffness parameters to predict all-cause mortality was compared. Additionally, we calculated the continuous net reclassification improvement and integrated discrimination improvement to compare the contribution of both parameters for all-cause mortality. A Cox-proportional hazards analyses were performed to identify the contributors to allcause mortality, and result is expressed as hazard ratio (HR) with a 95\% confidence interval (CI). A two-sided $\mathrm{P}$ value of 0.05 was considered statistically significant. Statistical analyses were performed using the software EZR (version 1.40, Saitama Medical Center, Jichi Medical University, Saitama, Japan). ${ }^{22}$

\section{Results}

\section{Comparison Identifying Variables Associated with All-Cause Mortality}

The baseline characteristics and the clinical features of patients who were alive and those who died during the 6 consecutive years of follow-up were compared (Table 1). Compared with alive patients, patients who died were of a higher age, CTR, CAVI and haPWV, and of lower diastolic $\mathrm{BP}$, serum albumin, phosphate and CPP. Most of these factors were then used as confounders in the Coxproportional hazards analyses shown in Figure 1 and Table 2.

\section{Causes of Death of Participants}

Of the 209 patients followed up, 38 (18.2\%) died. Cerebral stroke was the most common cause of death $(23.7 \%)$, followed by heart failure (15.7\%), coronary events $(13.7 \%)$, pneumonia $(7.7 \%)$ and disdialysis syndrome $(7.7 \%)$. The
Table I Comparison Identifying Variables Associated with AllCause Mortality

\begin{tabular}{|l|c|c|c|}
\hline & Alive & Dead & \multirow{2}{*}{ P value } \\
\cline { 2 - 3 } & $\mathbf{( N = I 7 I )}$ & $\mathbf{( N = 3 8 )}$ & \\
\hline Male (\%) & 60.8 & 65.8 & $0.46 I^{*}$ \\
Age (years) & $58.9 \pm 10.7$ & $70.3 \pm 11.3$ & $<0.001$ \\
Age $\geq 65$ years (\%) & 31 & 65.8 & $<0.00 I^{*}$ \\
HD vintage (year) & $7.9 \pm 6.9$ & $7.5 \pm 6.4$ & 0.843 \\
BMI (kg/m ${ }^{2}$ ) & $21.2 \pm 2.5$ & $20.6 \pm 2.7$ & 0.162 \\
BMI $\geq 25 \mathrm{~kg} / \mathrm{m}^{2}(\%)$ & 5.8 & 7.9 & $0.709^{*}$ \\
Systolic BP (mmHg) & $149 \pm 19$ & $147 \pm 21$ & 0.822 \\
Diastolic BP (mmHg) & $81 \pm 10$ & $76 \pm 10$ & 0.040 \\
CTR (\%) & $50.4 \pm 4.6$ & $53.7 \pm 6.2$ & $0.00 I^{*}$ \\
CAVI & $8.64 \pm 1.32$ & $9.64 \pm 1.47$ & $<0.001$ \\
haPWV (m/sec) & $8.60 \pm 1.16$ & $9.22 \pm 1.39$ & 0.005 \\
Hypertension (\%) & 70.8 & 68.4 & 0.845 \\
Albumin (g/dL) & $3.92 \pm 0.25$ & $3.79 \pm 0.29$ & 0.002 \\
Albumin <3.5 g/dL & 5.8 & 10.5 & $0.290^{*}$ \\
(\%) & & & \\
HbAlc (\%) & $5.24 \pm 0.68$ & $5.36 \pm 0.80$ & 0.202 \\
Diabetes (\%) & 24.6 & 34.2 & $0.227^{*}$ \\
TG (mg/dL) & $119 \pm 59$ & $107 \pm 53$ & 0.122 \\
TG $\geq 150 \mathrm{mg} / \mathrm{dL}(\%)$ & 21.1 & 18.4 & $0.827^{*}$ \\
HDL-C (mg/dL) & $50 \pm 15$ & $49 \pm 18$ & 0.608 \\
HDL-C < 40 mg/dL & 26.3 & 26.3 & $1.000^{*}$ \\
(\%) & & & \\
LDL-C (mg/dL) & $99 \pm 27$ & $100 \pm 31$ & 0.722 \\
LDL-C $\geq 120 \mathrm{mg} / \mathrm{dL}$ & 20.5 & 28.9 & $0.28 I^{*}$ \\
(\%) & & & \\
Albumin-adjusted & $9.2 \pm 0.7$ & $9.0 \pm 0.7$ & 0.427 \\
calcium (mg/dL) & & & \\
Phosphate (mg/dL) & $5.7 \pm 1.0$ & $5.3 \pm 0.9$ & 0.042 \\
CPP (mg $\left./ \mathrm{dL}{ }^{2}\right)$ & $52.5 \pm 11.4$ & $47.8 \pm 9.6$ & 0.026 \\
\hline
\end{tabular}

Notes: Data are presented as mean \pm SD. $P$ values were obtained using MannWhitney's U-test or *Fisher's exact test.

Abbreviations: HD, hemodialysis; BMI, body mass index; BP, blood pressure; CTR, cardio-thoracic ratio; CAVI, cardio-ankle vascular index; haPWV, heart-ankle pulse wave velocity; HbAlc, glycated hemoglobin; TG, triglyceride; HDL-C, high-density lipoprotein cholesterol; LDL-C, low-density lipoprotein cholesterol; CPP, calcium phosphate product.

less frequent causes included asphyxia (5.7\%), disseminated intravascular coagulation $(2.7 \%)$, sepsis $(2.7 \%)$, peripheral artery disease $(2.7 \%)$, femoral fracture $(2.7 \%)$, liver failure $(2.7 \%)$ and gastrointestinal bleeding $(2.7 \%)$. Deaths of unknown cause also accounted for $10.7 \%$.

\section{Pearson Product-Moment Correlation Coefficient Matrix (Color Coded)}

The correlations between clinical variables and arterial stiffness parameters were verified in Figure 2. In simple linear regression analyses, CAVI was positively correlated 
with male sex, age, systolic BP and $\mathrm{HbA1c}$, and negatively correlated with BMI and CPP. The association with systolic BP seemed to be greater for haPWV than for CAVI [Pearson's correlation coefficient (95\% CI): CAVI 0.269 (0.138-0.390) vs haPWV $0.618(0.527-0.696)]$.

\section{Survival Curves for the Unadjusted and Covariate-Adjusted Longitudinal Association of the Tertiles of CAVI and haPWV with All-Cause Mortality}

Figure 1 shows the event-free survival of all-cause mortality. Patients were divided into the tertile of CAVI (A, B) and haPWV (C, D). In addition, covariate-adjusted survival curves were estimated from the Cox-proportional hazards model, including age, albumin, CPP and diastolic $\mathrm{BP}$ as confounders (B, D).

There were significant differences among the tertile groups; the cumulative incidence of deaths was higher in highest tertile (T3) compared to T1/T2 for both CAVI and haPWV. The significant predictability of the arterial
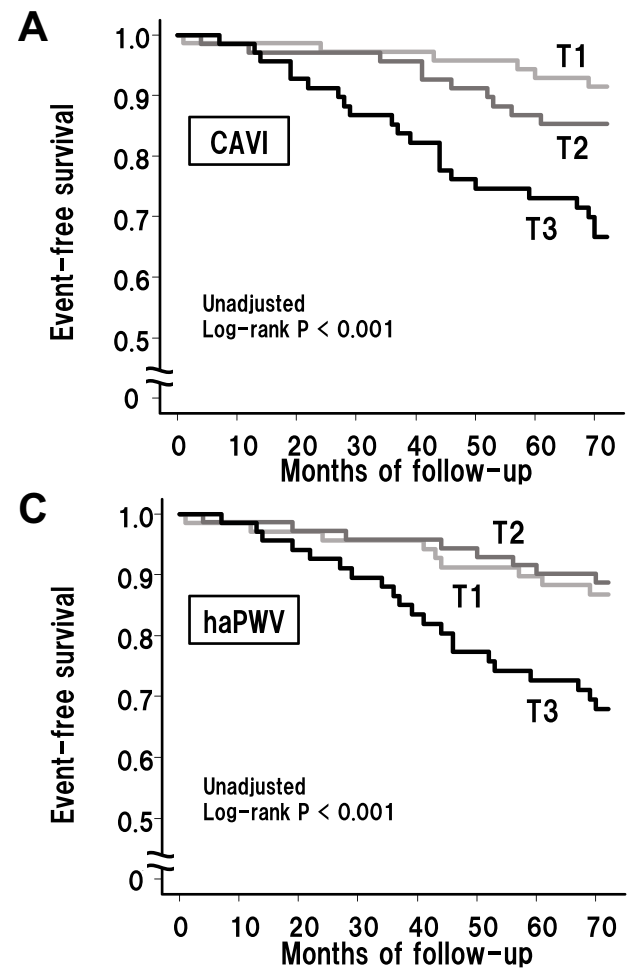

stiffness parameters on all-cause mortality were maintained after Cox-proportional hazards analyses.

\section{The ROC Curves and Youden's J Index}

Figure 3 shows the discriminatory powers of arterial stiffness parameters for the prediction of all-cause mortality.

The diagnostic accuracy of each arterial stiffness parameter (AUC, 95\% CI) for all-cause mortality was 0.698 (0.605-0.791) for CAVI, and $0.647(0.545-0.749)$ for haPWV. A significant difference was observed in the AUC between CAVI and haPWV $(\mathrm{P}=0.042)$. Furthermore, comparing the contribution of CAVI to allcause mortality with haPWV, a category-free net reclassification index was 0.573 (95\% CI $0.235-0.911, \mathrm{P}<0.001)$ and an integrated discrimination gain was $0.040(95 \% \mathrm{CI}$ 0. 019-0.060, $\mathrm{P}<0.001$ ), indicating the significant difference in both parameters. Additionally, the ROC curve analysis identified the cut-off values of both arterial stiffness parameters in predicting all-cause mortality as follows: 9.15 for CAVI, and 8.90 for haPWV. These cut-off
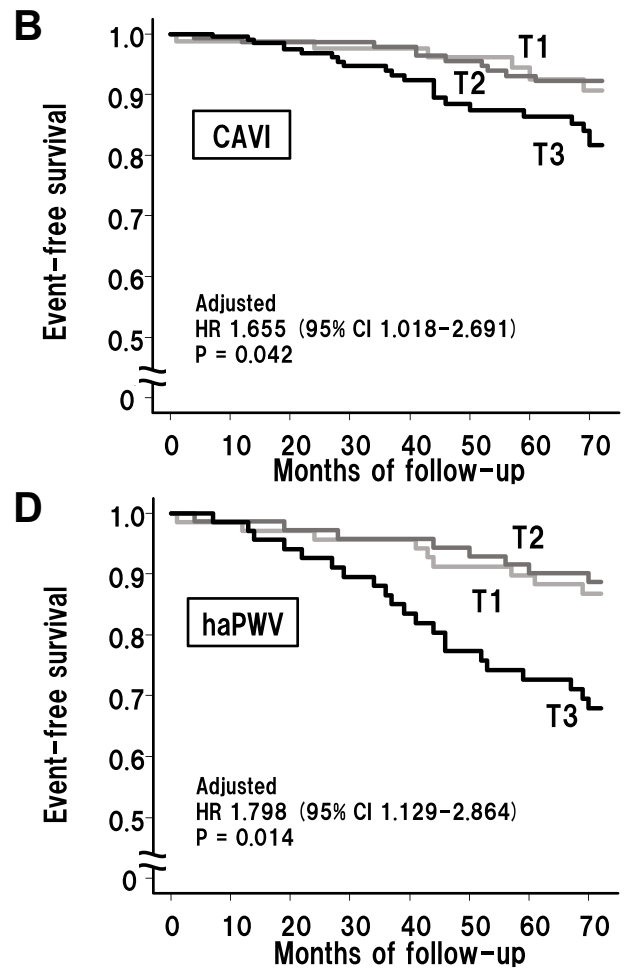

Figure I Survival curves for the unadjusted and covariate-adjusted longitudinal association of the tertiles of heart-ankle pulse wave velocity (haPWV) and cardio-ankle vascular index (CAVI) with all-cause mortality. Patients stratified by tertile of (A and B) CAVI and (C and $\mathbf{D})$ haPWV values. (B and $\mathbf{D})$ Covariate-adjusted survival curves estimated from the Cox-proportional hazards model, including age, albumin, CPP and diastolic BP as confounders.

Abbreviations: TI, lowest tertile; T2, middle tertile; T3, highest tertile. 
values were used in Cox-proportional hazards analyses shown in Table 2.

\section{Cox-Proportional Hazards Analyses for All-Cause Mortality}

The contribution of each arterial stiffness parameter to allcause mortality was examined using the Cox-proportional hazards analysis (Table 2). Because of the intraclass correlation between CAVI and haPWV, they were not used in the same model. Consequently, increases in the two parameters above the cut-off or every $1 \mathrm{SD}$ were independent predictors for all-cause mortality. In addition, age and CTR also contributed to all-cause mortality, whereas serum albumin, CPP, and diastolic BP did not.

\section{Discussion}

In order to clarify the role of arterial stiffness as a predictor of mortality rate of HD patients, CAVI which reflects the stiffness of the arterial tree from the origin of the aorta to the ankle was measured in 209 HD patients. During 6 consecutive years of follow-up, 38 patients (18\%) died. Patients who died had higher age, CTR, CAVI, haPWV, and lower diastolic BP, serum albumin and CPP compared to alive patients. In the Cox-proportional hazards analyses, 1 SD increase in both arterial stiffness parameters contributed independently to all-cause mortality, as well as age and CTR. Both parameters above the cut-offs estimated in the ROC analysis (CAVI $\geq 9.2$, haPWV $\geq 8.9$ ) also had independent contributions to mortality, although serum albumin and CPP did not. The Kaplan-Meier curves for cumulative survival among the tertile groups showed that the mortality rate was higher in highest tertile (T3) compared to T1/T2 for both CAVI and haPWV. These results suggested that CAVI could be a predictor for all-cause mortality in HD patients.

In the present study, although haPWV was also shown to be a predictor for the mortality, ROC analysis showed that CAVI had better discriminatory power for all-cause mortality compared to haPWV. This result seems to be inconsistent to the findings of Kato et $\mathrm{al}^{19}$ using baPWV. The two studies have different subjects and observation conditions, and it is still unclear why the results are different. In addition, the result of ROC analysis does not necessarily guarantee a more effective clinical relevance of CAVI. Based on these shortcomings, large-scale prospective cohort studies are needed in the future.

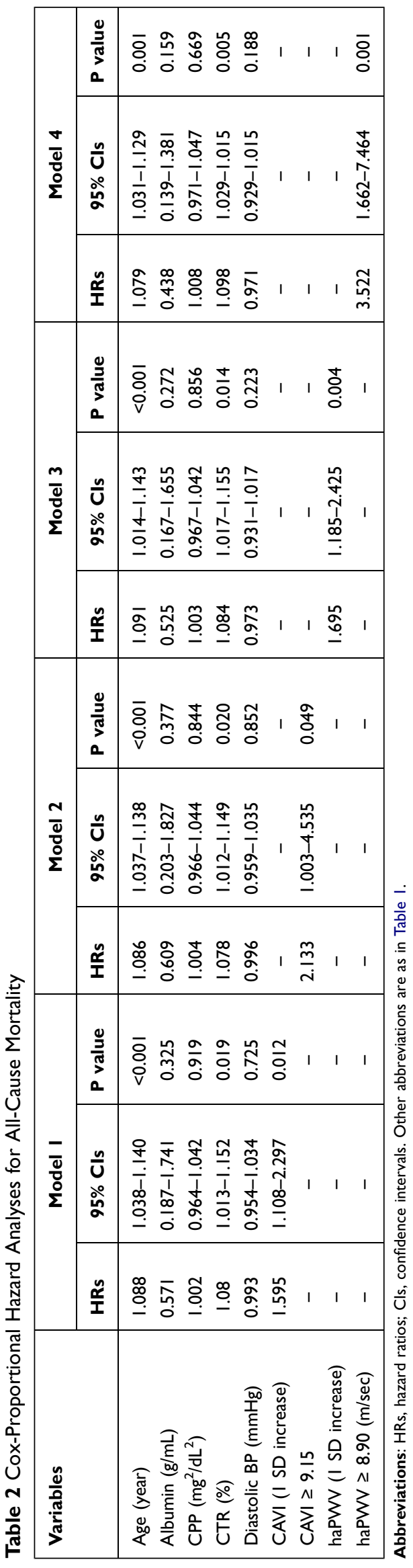




\begin{tabular}{|c|c|c|c|c|c|c|c|c|c|c|c|c|c|c|}
\hline CAVI & & 0.904 & 0.180 & 0.398 & -0.118 & 0.088 & 0.269 & -0.045 & -0.194 & 0.242 & -0.089 & -0.073 & -0.104 & -0.306 \\
\hline haPWV & 0.904 & & 0.181 & 0.182 & -0.214 & 0.028 & 0.618 & 0.315 & -0.100 & 0.304 & -0.012 & -0.095 & -0.095 & -0.201 \\
\hline Male & 0.180 & 0.181 & & -0.045 & -0.008 & -0.207 & 0.097 & 0.039 & 0.120 & 0.143 & -0.062 & -0.317 & -0.238 & -0.068 \\
\hline Age & 0.398 & 0.182 & -0.045 & & -0.071 & 0.375 & -0.213 & -0.392 & -0.207 & -0.062 & $\mid-0.109$ & -0.120 & 0.060 & -0.409 \\
\hline HD vintage & -0.118 & -0.214 & -0.008 & -0.071 & & 0.068 & -0.280 & -0.205 & -0.177 & -0.235 & -0.097 & 0.084 & 0.007 & 0.212 \\
\hline CTR & 0.088 & 0.028 & -0.207 & 0.375 & 0.068 & & -0.048 & -0.114 & -0.044 & -0.043 & 0.041 & -0.077 & 0.112 & 0.009 \\
\hline SBP & 0.269 & 0.618 & 0.097 & -0.213 & -0.280 & -0.048 & & 0.620 & 0.061 & 0.273 & 0.118 & -0.088 & -0.012 & 0.082 \\
\hline DBP & -0.045 & 0.315 & 0.039 & -0.392 & -0.205 & -0.114 & 0.620 & & 0.211 & 0.121 & 0.110 & -0.068 & -0.015 & 0.126 \\
\hline BMI & -0.194 & -0.100 & 0.120 & -0.207 & -0.177 & -0.044 & 0.061 & 0.211 & & 0.130 & 0.367 & -0.351 & 0.003 & 0.202 \\
\hline $\mathrm{HbA1c}$ & 0.242 & 0.304 & 0.143 & -0.062 & -0.235 & -0.043 & 0.273 & 0.121 & 0.130 & & 0.153 & -0.159 & -0.019 & -0.123 \\
\hline$T G$ & -0.089 & -0.012 & -0.062 & -0.109 & -0.097 & 0.041 & 0.118 & 0.110 & 0.367 & 0.153 & & -0.419 & 0.222 & 0.160 \\
\hline$H D L-C$ & -0.073 & -0.095 & -0.317 & -0.120 & 0.084 & -0.077 & -0.088 & $\mid-0.068$ & -0.351 & -0.159 & -0.419 & & 0.077 & 0.031 \\
\hline LDL-C & \begin{tabular}{|l|}
-0.104 \\
\end{tabular} & -0.095 & -0.238 & 0.060 & 0.007 & 0.112 & -0.012 & -0.015 & 0.003 & -0.019 & 0.222 & 0.077 & & 0.066 \\
\hline CPP & -0.306 & -0.201 & -0.068 & -0.409 & 0.212 & 0.009 & \begin{tabular}{|l|}
0.082 \\
\end{tabular} & \begin{tabular}{|l|}
0.126 \\
\end{tabular} & 0.202 & -0.123 & 0.160 & 0.031 & 0.066 & \\
\hline & $\underset{\mathrm{J}}{\mathbf{J}}$ & 彥 & 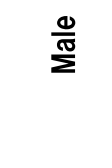 & $\stackrel{8}{8}$ & 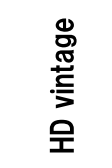 & 品 & 愛 & 僉 & $\bar{\Sigma}$ & $\frac{0}{\frac{0}{0}}$ & $\stackrel{5}{\circ}$ & $\begin{array}{l}\text { ㅁ } \\
\text { 무 }\end{array}$ & 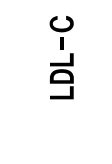 & 몽 \\
\hline
\end{tabular}

Figure 2 Pearson product-moment correlation coefficient matrix. Pearson's correlation coefficients are listed in the appropriate section in the matrix. The sections are color-coded according to the degree of correlation.

There are several reasons to explain why high CAVI is related to a high mortality rate in HD patients. First, it is mentioned that the severity of atherosclerosis is directly related to the occurrence of CVD events. In fact, Kirigaya et al reported that CAVI is an independent long-term predictor of major adverse CVD events, especially CVD death. ${ }^{17}$ Second, it is known that the correlation between heart function and CAVI exists. Zhang et $\mathrm{al}^{23}$ reported that heart failure patients show a high CAVI, and improvement of heart failure (elevated left ventricular ejection fraction) was associated with a decrease in CAVI. These results indicate that high CAVI causes heart failure to deteriorate. Therefore, high CAVI in HD patients might be one of the causes of mortality due to deteriorating heart failure. Third, sarcopenia and/or frailty were significantly associated with mortality for all age strata in men and women (except in women aged 37-45 years) after adjustment for confounders. ${ }^{24}$ CAVI was reported to be high in subjects with sarcopenia, ${ }^{25,26}$ suggesting that high CAVI might be related to high mortality through sarcopenia and/or frailty.

Generally, diabetes mellitus, dyslipidemia, elevated BP and lack of exercise are considered to be high risks for CAVI. ${ }^{27}$ In the present study, as shown in Figure 2, age, systolic BP, HbA1c and CPP were related to CAVI in simple regression analysis. Therefore, hypertension and diabetes mellitus must be carefully treated in HD patients. It has already been reported that CAVI can be decreased by appropriate treatments and behavior modification. ${ }^{27}$ In addition to the management of metabolic disorders, smoking cessation, ${ }^{28}$ treatment of periodontitis, ${ }^{29}$ improvement of sleep duration, ${ }^{30}$ and correction of sleep apnea ${ }^{31}$ may contribute to the improvement of CAVI. Accordingly, HD patients with high CAVI may be able to reduce the risk of mortality through the reduction of CAVI by therapeutic approaches considering the cause.

In the present study, CTR was an independent predictor to all-cause mortality in HD patients. This finding is consistent with the previous report indicating that CTR can be used for CVD risk stratification in HD patients. ${ }^{32}$ Therefore, the combination of the arterial stiffness parameter and CTR may lead to greater accuracy in the assessment of mortality risk in HD patients.

High serum phosphorus is generally reported to be related to mortality in HD patients. ${ }^{1,20}$ However, the present study showed that serum phosphorus level and CPP were rather low in the patients who died. Also, in this 


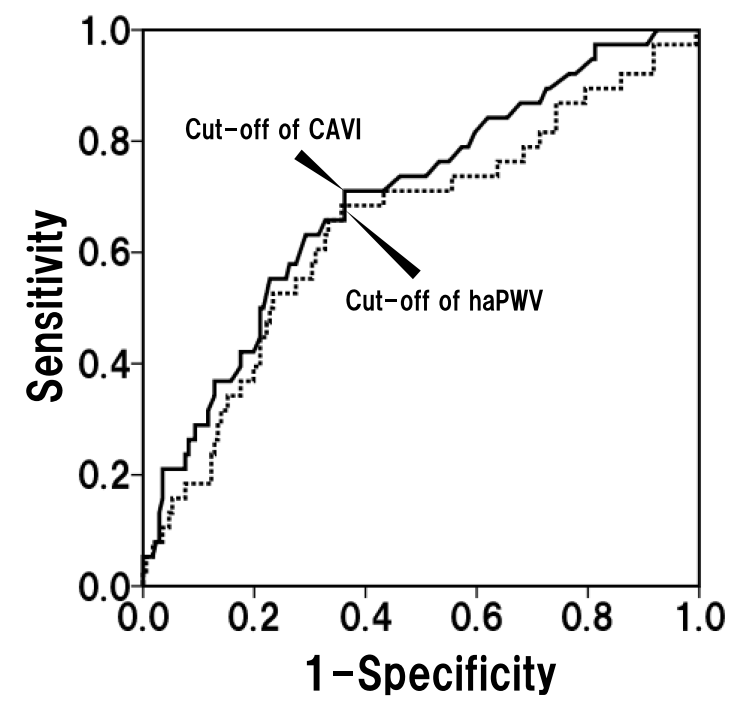

\begin{tabular}{|c|c|c|c|c|c|c|c|}
\hline Line & & Cut-off & Sensitivity & Specificity & AUC $(95 \% \mathrm{Cl})$ & NRI $(95 \% \mathrm{Cl})$ & IDI $(95 \% \mathrm{Cl})$ \\
\hline$\ldots \ldots$. & : haPWV & 8.90 & $68.4 \%$ & $64.3 \%$ & $0.647(0.544-0.750)$ & - & - \\
\hline & : CAVI & 9.15 & $71.1 \%$ & $63.7 \%$ & $0.698 *(0.605-0.791)$ & $0.573^{\dagger}(0.235-0.911)$ & $0.040^{+}(0.019-0.060)$ \\
\hline
\end{tabular}

Figure 3 Discriminatory powers of arterial stiffness parameters for the prediction of all-cause mortality. Curves represent receiver-operating-characteristics (ROC) analyses for discriminating the probability of all-cause mortality. The Youden Index was used to select the optimum cut-off point of each arterial stiffness parameter. $* \mathrm{P}<$ $0.05,{ }^{\dagger} \mathrm{P}<0.00 \mathrm{l}$ between haPWV and CAVI.

Abbreviations: AUC, area under curve; NRI, net reclassification index; IDI, integrated discrimination improvement; $95 \% \mathrm{Cl}$, $95 \%$ confidence interval; haPWV, heart-ankle pulse wave velocity; CAVI, cardio-ankle vascular index.

study, CPP was rather negatively correlated with CAVI. The paradox that CPP, a measure of disturbed mineral metabolism, is associated with reduced arterial stiffness needs to be further explored.

\section{Limitations}

The limitations of the present study are as follows: the number of HD patients who were followed in the 2 institutions managing HD included only a small number, and this was a retrospective study.

\section{Conclusion}

Through the 6 consecutive years of follow-up in 209 HD patients, increased CAVI might represent a major modifiable risk factor for all-cause mortality. Further research is needed to examine whether CAVI-lowering interventions contribute to improved prognosis.

\section{Funding}

There is no funding to report.

\section{Disclosure}

The authors have no conflicts of interest to declare.

\section{References}

1. Ma L, Zhao S. Risk factors for mortality in patients undergoing hemodialysis: a systematic review and meta-analysis. Int $J$ Cardiol. 2017;238:151-158. doi:10.1016/j.ijcard.2017.02.095

2. Go AS, Chertow GM, Fan D, McCulloch CE, Hsu CY. Chronic kidney disease and the risks of death, cardiovascular events, and hospitalization. $N$ Engl J Med. 2004;351:1296-1305. doi:10.1056/ NEJMoa041031

3. Nitta K, Goto S, Masakane I, et al.; on behalf of the Japanese Society for Dialysis Therapy Renal Data Registry Committee. Annual dialysis data report for 2018, JSDT Renal Data Registry: survey methods, facility data, incidence, prevalence, and mortality. Ren Replace Ther. 2020;6:41. doi:10.1186/s41100-020-00286-9

4. Imanishi R, Seto S, Toda G, et al. High brachial-ankle pulse wave velocity is an independent predictor of the presence of coronary artery disease in men. Hypertens Res. 2004;27:71-78. doi:10.1291/hypres.27.71

5. El Ghoul B, Daaboul Y, Korjian S, et al. Etiology of end-stage renal disease and arterial stiffness among hemodialysis patients. Biomed Res Int. 2017;2017:2543262. doi:10.1155/2017/2543262

6. Shirai K, Utino J, Otsuka K, Takata M. A novel blood pressure independent arterial wall stiffness parameter; cardio-ankle vascular index (CAVI). J Thromb. 2006;13:101-107.

7. Hayashi K, Handa H, Nagasawa S, Okumura A, Moritake K. Stiffness and elastic behavior of human intracranial and extracranial arteries. J Biomech. 1980;13:175-184. doi:10.1016/0021-9290(80)90191-8

8. Kawasaki T, Sasayama S, Yagi S, Asakawa T, Hirai T. Non-invasive assessment of the age related changes in stiffness of major branches of the human arteries. Cardiovasc Res. 1987;21:678-687. doi:10.1093/ $\mathrm{cvr} / 21.9 .678$

9. Bramwell JC, Hill AV. The velocity of the pulse wave in man. Proc $R$ Soc London Series B. 1926;93:298-306. 
10. Shirai K, Song M, Suzuki J, et al. Contradictory effects of $\beta 1$ - and $\alpha 1$-aderenergic receptor blockers on cardio-ankle vascular stiffness index (CAVI) - The independency of CAVI from blood pressure. $J$ Atheroscler Thromb. 2011;18:49-55. doi:10.5551/jat.3582

11. Shirai K, Suzuki K, Tsuda S, Shimizu K, Takata M, Yamamoto T. Comparison of cardio-ankle vascular index (CAVI) and CAVI0 in large healthy and hypertensive populations. $J$ Atheroscler Thromb. 2019;26:603-615. doi:10.5551/jat.48314

12. Nakamura K, Iizuka T, Takahashi M, et al. Association between cardio-ankle vascular index and serum cystatin $\mathrm{C}$ levels in patients with cardiovascular risk factor. $J$ Atheroscler Thromb. 2009;16:371-379. doi:10.5551/jat.No687

13. Kubozono T, Miyata H, Uegama K, et al. Association between arterial stiffness and estimated glomerular filtration rate in the Japanese general population. $J$ Atheroscler Thromb. 2009;16:840-845. doi:10.5551/jat.1230

14. Ueyama K, Miyata M, Kubozono T, et al. Noninvasive indices of arterial stiffness in hemodialysis patients. Hypertens Res. 2009;32:716-720. doi:10.1038/hr.2009.83

15. Ichihara A, Yamashita N, Takemitsu T, et al. Cardio-ankle vascular index and ankle pulse wave velocity as a marker of arterial fibrosis in kidney failure treated by hemodialysis. Am $J$ Kidney Dis. 2008;52:947-955. doi:10.1053/j.ajkd.2008.06.007

16. Sato Y, Nagayama D, Saiki A, et al. Cardio-ankle vascular index is independently associated with future cardiovascular events in outpatients with metabolic disorders. $J$ Atheroscler Thromb. 2016;23:596-605. doi:10.5551/jat.31385

17. Kirigaya J, Iwahashi N, Tahakashi H, et al. Impact of cardio-ankle vascular index on long term outcome in patients with acute coronary syndrome. J Atheroscler Thromb. 2020;27:657-668. doi:10.5551/ jat.51409

18. Itano $\mathrm{S}$, Yano $\mathrm{Y}$, Nagasu $\mathrm{H}$, et al. Association of arterial stiffness with kidney function among adults without chronic kidney disease. Am J Hypertens. 2020;33:1003-1010. doi:10.1093/ajh/hpaa097

19. Kato A, Takita T, Furuhashi M, Maruyama Y, Miyajima H, Kumagai H. Brachial-ankle pulse wave velocity and the cardio-ankle vascular index as a predictor of cardiovascular outcomes in patients on regular hemodialysis. Ther Apher Dial. 2012;16:232-241. doi:10.1111/j.1744-9987.2012.01058.x

20. Lopes MB, Karaboyas A, Bieber B, et al. Impact of longer-term phosphorus control on cardiovascular mortality in hemodialysis patients using an area under the curve approach: results from the DOPPS. Nephrol Dial Transplant. 2020;35:1794-1801. doi:10.1093/ $\mathrm{ndt} / \mathrm{gfaa} 054$
21. Youden WJ. Index for rating diagnostic tests. Cancer. 1950;3:32-35. doi:10.1002/1097-0142(1950)3:1<32::AID-CNCR2820030106>3.0. $\mathrm{CO} ; 2-3$

22. Kanda Y. Investigation of the freely available easy-to-use software 'EZR' for medical statistics. Bone Marrow Transplant. 2013;48:452-458. doi:10.1038/bmt.2012.244

23. Zhang C, Ohira M, Iizuka T, et al. Cardio-ankle vascular index relates to left ventricular ejection fraction in patients with heart failure. A retrospective study. Int Heart J. 2013;54(4):216-221. doi:10.1536/ihj.54.216

24. Hanlon P, Nicholl BI, Jani BD, Lee D, McQueenie R, Mair FS. Frailty and pre-frailty in middle-aged and older adults and its association with multimorbidity and mortality: a prospective analysis of 493737 UK Biobank participants. Lancet Public Health. 2018;3: e323-e332. doi:10.1016/S2468-2667(18)30091-4

25. Kirkham FA, Bunting E, Fantin F, Zamboni M, Rajkumar C. Independent association between cardio-ankle vascular index and sarcopenia in older U.K. adults. J Am Geriatr Soc. 2019;67 (2):317-322. doi:10.1111/jgs.15648

26. Ogawa A, Shimizu K, Nakagami T, Maruoka H, Shirai K. Physical function and cardio-ankle vascular index in elderly heart failure patients. Int Heart J. 2020;61:769-775. doi:10.1536/ihj.20-058

27. Saiki A, Ohira M, Yamaguchi T, et al. New horizons of arterial stiffness developed using cardio-ankle vascular index (CAVI). $J$ Atheroscler Thromb. 2020;27:732-748. doi:10.5551/jat.RV17043

28. Noike H, Nakamura K, Sugiyama Y, et al. Changes in cardio-ankle vascular index in smoking cessation. $J$ Atheroscler Thromb. 2010;17 (5):517-525. doi:10.5551/jat.3707

29. Hayashida H, Saito T, Kawasaki K, et al. Association of periodontitis with carotid artery intima-media thickness and arterial stiffness in community-dwelling people in Japan: the Nagasaki Islands study. Atherosclerosis. 2013;229(1):186-191. doi:10.1016/j. atherosclerosis.2013.04.002

30. Morita N, Kambayashi I, Okuda T, et al. Inverse relationship between sleep duration and cardio-ankle vascular index in children. J Atheroscler Thromb. 2017;24(8):819-826. doi:10.5551/jat.36517

31. Tomita Y, Kasai T. Relationship between cardio-ankle vascular index and obstructive sleep apnea. Rev Cardiovasc Med. 2020;21 (3):353-363. doi:10.31083/j.rcm.2020.03.67

32. Okute Y, Shoji T, Hayashi T, et al. Cardiothoracic ratio as a predictor of cardiovascular events in a cohort of hemodialysis patients. J Atheroscler Thromb. 2017;24(4):412-421. doi:10.5551/jat.36426
Vascular Health and Risk Management

\section{Publish your work in this journal}

Vascular Health and Risk Management is an international, peerreviewed journal of therapeutics and risk management, focusing on concise rapid reporting of clinical studies on the processes involved in the maintenance of vascular health; the monitoring, prevention and treatment of vascular disease and its sequelae; and the involvement of metabolic disorders, particularly diabetes. This journal is indexed on PubMed Central and MedLine. The manuscript management system is completely online and includes a very quick and fair peerreview system, which is all easy to use. Visit http://www.dovepress. com/testimonials.php to read real quotes from published authors. 